\title{
Detection of Shockable Rhythms Using Convolutional Neural Networks During Chest Compressions Provided by a Load Distributing Band
}

\author{
Iraia Isasi ${ }^{1}$, Unai Irusta ${ }^{1}$, Elisabete Aramendi $^{1}$, Jan-Åge Olsen ${ }^{2}$, Lars Wik $^{2,3}$ \\ ${ }^{1}$ Communications Engineering, University of the Basque Country (UPV/EHU), Bilbao, Spain \\ ${ }^{2}$ Oslo University Hospital, Oslo, Norway \\ ${ }^{3}$ Norwegian National Advisory Unit on Prehospital Emergency Medicine, Oslo, Norway
}

\begin{abstract}
Load Distributing Band (LDB) mechanical chest compression devices are used to treat out-of-hospital cardiac arrest (OHCA) patients. The artefacts that $L D B$ chest compressions induce in the ECG impede a reliable shock/noshock diagnosis, resulting in compression interruptions to analyze the ECG. The aim of this study was to design a deep learning algorithm to accurately detect shockable rhythms with concurrent LDB compressions. The dataset was comprised of 780 shockable and 2644 nonshockable rhythms from 242 OHCA patients treated with the LDB device. Underlying rhythms were annotated by expert reviewers in artefact-free intervals. The method consisted of two stages: a Recursive Least Squares (RLS) filter to remove $L D B$ compression artefacts and a shock/no-shock decision algorithm based on CNNs. Shock/no-shock diagnoses were compared with the rhythm annotations to obtain the sensitivity $(\mathrm{Se})$ and specificity $(\mathrm{Sp})$ of the method. The median Se, Sp were 92.2\%, 96.6\%, respectively. The proposed algorithm met the American Heart Association's (AHA) requirements for rhythm analysis.
\end{abstract}

\section{Introduction}

Early defibrillation together with high quality cardiopulmonary resuscitation (CPR) is critical to survival from out-of-hospital cardiac arrest (OHCA) [1]. Defibrillation is achieved through an electrical shock administered by a defibrillator, an electromedical device that incorporates a shock advice algorithm (SAA) that analyzes the ECG to detect shockable rhythms. Unfortunately, the mechanical activity of the chest compressions induces artefacts in the ECG that impede a reliable shock advice [2,3]. Therefore, current defibrillators intruct the rescuer to stop CPR to analyze the rhythm on artefact-free ECG. These recurring "hands-off" intervals lead to an intermittent lack of cerebral and myochardial blood flow, which reduces the probability of OHCA survival [1].
Several mechanical chest compression devices have been introduced in the OHCA setting to optimize the delivery of CPR $[4,5]$. Although they are not associated to improved survival, the use of mechanical devices is strongly recommended in pronloged CPR and in scenarios where manual chest compressions are impractical or compromise rescuer safety [1]. Their use has increased drastically in the last two decades. There are two types of automated compressors: pneumatically driven pistons like the LUCAS (Physio-Control Inc/Jolife, AB, Lund, Sweeden), and Load Distributing Bands (LDB) like the AutoPulse (Zoll Circulation, Chelmsford, Massachusetts, USA). Since the use of these devices is becoming commonplace, a reliable rhythm analysis during mechanical CPR is critical. In this way, "hands-off" intervals would be minimized improving the probability of OHCA survival. SAAs compliant with the accuracy requirements of the American Heart Association (AHA) have already been demonstrated during pistondriven chest compressions [6]. These SAAs consist of two stages: an adaptive filter to remove compression artefacts followed by a shock/no-shock decision algorithm based on traditional machine learning techniques. However, a reliable rhythm analysis during the usage of LDB devices has not yet been demonstrated.

In the context of supervised learning, deep learning (DL) techniques have proven to be very efficient for biomedical signal classification tasks, contributing to important advances even in the field of OHCA. In particular, recent studies show that deep learning techniques outperform tradiditional machine learning algorithms in the shock/no-shock diagnosis of the rhythm during intervals free of chest compressions [7-10] and during manual CPR [11].

Following this line, the aim of this work was to design a DL-based algorithm for the discrimination of shockable and nonshockable rhythms during CPR provided by a LDB device. The method comprises two stages: an adaptive RLS filter to remove LDB compression artefacts, followed by a shock/no-shock decision algorithm based on Convo- 
lutional Neural Networks (CNN).

\section{Materials and methods}

\subsection{Materials}

The data used in this study were extracted from a randomized control trial (Circulation Improving Resuscitation Care, CIRC) which aimed to compare the effectiveness of CPR provided by the AutoPulse device against manually administered CPR in terms of survival [4]. The cardiac arrest episodes were collected by the emergency services of three American (The Fox Valley, Hilssborough, Houston) and two European sites (Vienna, Nijmegen) between March 2009 and January 2011. The ECG and thoracic impedance (TI) signals acquired using the Lifepak 12 or 15 monitor-defibrillator (Physio Control, Redmond, WA, USA) were exported to Matlab and resampled to $250 \mathrm{~Hz}$ for processing.

The dataset extracted from these episodes consists of 3424 segments of $22 \mathrm{~s}$ from 242 OHCA patients treated with the LDB device. The segments include an interval of $16 \mathrm{~s}$, during chest compressions, used to develop our solution, followed or preceded by an interval of $6 \mathrm{~s}$, free of artefacts, used to annotate the underlying rhythm of the patient. Fig 1 shows two examples from the study dataset corresponding to a patient in a nonshockable rhythm (panel a) and to a patient in a shockable rhythm (panel b). The final dataset consists of 2644 nonshockable rhythms (asystole, sinus normal and other regular rhythms) and 780 shockable rhythms (ventricular fibrilation and rapid ventricular tachycardia).

\subsection{Methods}

The SAA proposed in this study is composed of two stages. First, an adaptive RLS filter is used to remove chest compression artefacts from the ECG. Then, a shock/noshock decision algorithm was designed to classify the filtered ECG using CNNs.

\section{CPR artefact suppressing filter}

Chest compressions provided by the LDB device have a constant rate of $80 \mathrm{~min}^{-1}\left(f_{0}=1.33 \mathrm{~Hz}\right)$ and a depth corresponding to $20 \%$ of the chest height. The pattern of the artefact is therefore quasi-periodic and can be represented as a Fourier series of $N$ harmonics locked to the frequency of the chest compressions, $f_{0}$ :

$$
s_{\mathrm{cpr}}(n)=\sum_{k=1}^{N} a_{k}(n) \cos \left(k \omega_{0} n\right)+b_{k}(n) \sin \left(k \omega_{0} n\right)
$$

where $\omega_{0}=2 \pi f_{0} T_{s}$ and $T_{s}$ is the sampling period.

The time-varying in-phase, $a_{k}(n)$, and quadrature, $b_{k}(n)$, coefficients were adaptively estimated using the Recursive Least Squares (RLS) algorithm [12] to minimize the error between the corrupt ECG, $s_{\mathrm{cor}}(n)$, and $s_{\mathrm{cpr}}(n)$ at the harmonics of $f_{0}$. The underlying rhythm was then obtained by substraction: $\hat{s}_{\mathrm{ecg}}(n)=s_{\mathrm{cor}}(n)-s_{\mathrm{cpr}}(n)$. The parameters governing the RLS filter, i.e. the number of harmonics $(N)$ and the forgetting factor $(\lambda)$, were respectively fixed to 31 and 0.988 according to [13].

\section{Shock/no-shock decision algorithm}

The shock/no-shock decision algorithm was designed to analyze the filtered ECG in the interval from $4-12$ s during chest compressions (see highlighted interval of Fig. 1), i.e. the algorithm used $8 \mathrm{~s}$ for a decision.

The deep learning algorithm designed in this study for the shock/no-shock decision consisted of two main blocks: a CNN to extract the high level descriptors of the ECG, and two fully connected layers for classification. The $8 \mathrm{~s}$
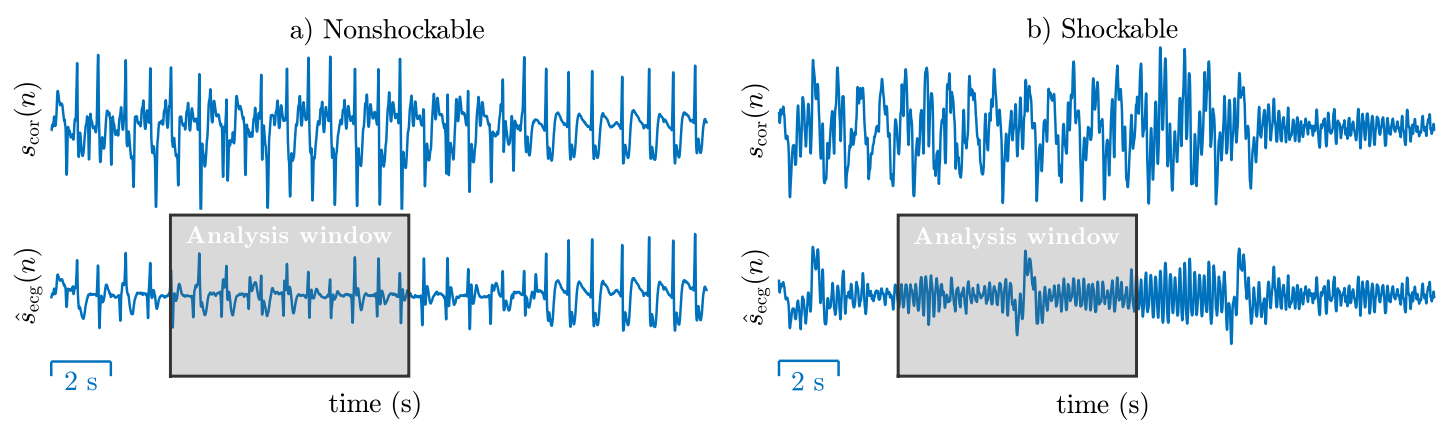

Figure 1. Two examples of $22 \mathrm{~s}$ ECG segments corresponding to a patient in a nonshockable rhythm (example a) and in a shockable rhythm (example b). The first panels show the corrupted ECG recorded by the defibrillator during mechanical chest compressions, $s_{\text {cor }}(n)$, whereas the second panels show the ECG after filtering the artefact, $\hat{s}_{\text {ecg }}(n)$. The first $16 \mathrm{~s}$ of both panels are corrupted by LDB compression artefacts whereas the last $6 \mathrm{~s}$ show the underlying rhythm of the patient. 
window of the filtered ECG was downsampled to $125 \mathrm{~Hz}$ before it was fed into the CNN, resulting in a 1-D signal of $N=1000$ samples.

The CNN was composed of $B$ convolutional blocks. The $b$-th block consisted of a convolutional layer that applied $J_{b}$ filters of width $I$ to the filtered ECG, followed by a batch normalization layer and a rectified linear unit (ReLU). So, denoting by $s_{b-1}(n, m)$ the output of block $b-1$ (input to block $b$ ) the output of the convolutional layer at block $b$ would be:

$$
c_{b}(n, m)=f\left(b_{m}+\sum_{\ell=1}^{J_{b-1}} \sum_{i=1}^{I_{b}} \omega_{\ell, i}^{m} s_{b-1}(n+i-1, \ell)\right)
$$

where $f(x)=\max (x, 0)$ is the ReLU activation function that makes the network non-linear, $n$ is the time index and $m$ the filter index. The input to the first block is the filtered ECG, that is, $s_{0}(n, 1)=\hat{s}_{\text {ecg }}(n)$.

A max-pooling layer of size $K=3$ was used at the end of each convolutional block to reduce by one third the dimensionality of the characteristics extracted by the CNN. Padding was applied at the output of each convolutional layer to preserve signal length, so the only reduction of the dimensionality occurred at the max-pooling layers. The features learned by the convolutional blocks were fed into two-fully-connected layers. The first one was composed of 10 hidden units whereas the second one used 2 neurons for the 2-class classification task. Finally, a softmax function was used to convert the output of the last two neurons into two values in the $[0,1]$ range that can be interpreted as the likelihood that the $8 \mathrm{~s}$ window is shockable or nonshockable.

The weights and biases of every layer corresponding to the classification stage were optimized using stochastic gradient descent with a momentum of 0.8 . The initial learning rate was fixed to 0.02 and it was reduced by a factor of 0.8 at every epoch. The training data were fed into the CNN in batches of 256, and 20 epochs were used to train the networks. In order to address class imbalance, the number of shockable segments was augmented by a factor of 1.5 during training.

\subsection{Evaluation}

Data were randomly partitioned patient-wise into training $(80 \%)$ and test $(20 \%)$ sets ensuring that shock/noshock case prevalences resembled those of the whole dataset. The shock/no-shock decisions of the SAA in the test set were compared with the clinicians' annotations for the artifact-free intervals to obtain the following performance metrics: sensitivity (Se), the proportion of correctly identified shockable segments; specificity (Sp), the proportion of correctly identified nonshockable segments and the balanced accuracy (BAC), i.e. the mean value of Se and Sp. The positive predictive value (PPV) and the total accuracy (Acc) were also computed as overall performance metrics. The process was repeated 100 times to statistically characterize these performance metrics.

\section{Results}

Fig. 2 shows the median values of the BAC obtained in 100 random repetitions of the test set as a function of the parameters governing $\mathrm{CNN}$ performance. Three parameters were studied: the number of blocks, $B$, the size of the filters $I$, and the number of filters, $\mathbf{L}=\left(J_{1}, \ldots J_{B}\right)$. As different number of filters were applied in each convolutional layer, the tested values were shrinked to four configurations (from dense to sparse): $\mathbf{L}_{\mathbf{4}}=(40,30,20,10), \mathbf{L}_{\mathbf{3}}=(32,24,16,8)$, $\mathbf{L}_{\mathbf{2}}=(24,18,12,6)$ and $\mathbf{L}_{\mathbf{1}}=(16,12,8,4)$. The number in parentheses indicate the amount of filters from block 1 to block 4 , so for an architecture with 3 blocks and $\mathbf{L}_{2}$ the architecture would be $(24,18,12)$. The best results were obtained for $B=3, I=8$ and a filter configuration of $\mathbf{L}_{\mathbf{3}}=(32,24,16)$. Adding a fourth block increased the complexity, i.e. the number of trainable parameters, and did not improve the results obtained for the best performing architecture. Using only 2 blocks lead to a drop of more than 1.5 points in BAC.

The median ( $90 \%$ confidence interval) values for Se, Sp, BAC, Acc and PPV obtained with the best performing architecture were 92.2\%(86.1-95.5), 96.6\% (94.4-98.0), $94.3 \%(91.3-95.9), 95.3 \%(93.7-96.8)$ and $89.0 \%(81.4-$ 93.8), respectively. These results meet the minimum $90 \%$ Se and $95 \% \mathrm{Sp}$ performance goals recommended by the AHA for artefact-free ECGs.

\section{Conclusions}

This work introduces the first AHA compliant shock/noshock decision algorithm with concurrent LDB chest compressions. It consists of an adaptive RLS filter designed to remove LDB compression artefacts and a shock/no-shock decision algorithm based on CNNs.

As shown in Fig. 2, the model starts to overfit when more than $3 \mathrm{CNN}$ blocks are used, as from then on the number of trainable parameters is too large for the size of the dataset. Adding more data would allow the design of deeper networks leading to the extraction of more sophisticated features and so, to better performance. Futhermore, more accurate SAAs have been demonstrated during manual chest compressions when the diagnosis is made using a majority criterion on three shorter consecutive intervals [11]. In brief, there is still room to improve the accuracy of rhythm analysis with concurrent LDB compressions, which may lead to accurate enough solutions that 

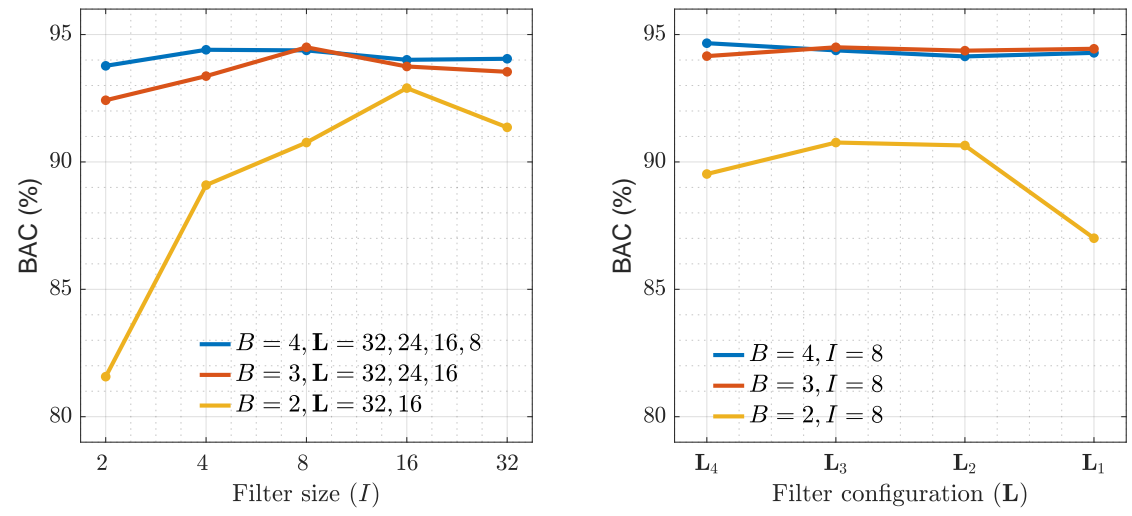

Figure 2. Performance of the SAA as a function of the configurable parameters in the CNN: number of blocks, $B$, the filter size, $I$, and the filter configuration, $\mathbf{L}$. The left panel shows the effect of the filter size for $\mathbf{L}_{3}$ whereas the right panel shows the effect of the different filter configurations, $\mathbf{L}_{\mathbf{1}}-\mathbf{L}_{\mathbf{4}}$, for $I=8$.

bring uninterrupted mechanical CPR into real practice.

\section{Acknowledgements}

This work was supported by the Spanish Ministerio de Ciencia, Innovacion y Universidades through grant RTI2018-101475-BI00, jointly with the Fondo Europeo de Desarrollo Regional (FEDER), and by the Basque Government through grants IT1229-19 and PRE-2019-2-0066.

\section{References}

[1] Perkins G, Handley A, Koster R, Castrén M, Smyth M, Olasveengen T, Monsieurs K, Raffay V, Gärsner J, Wenzel $\mathrm{V}$, et al. European resuscitation council guidelines for resuscitation 2015: Section 2. Adult basic life support and automated external defibrillation. Resuscitation 2015;95:81-99.

[2] Ruiz de Gauna S, Irusta U, Ruiz J, Ayala U, Aramendi E, Eftest $\varnothing 1 \mathrm{~T}$. Rhythm analysis during cardiopulmonary resuscitation: past, present, and future. BioMed Research International 2014;2014:Article ID 386010.

[3] Aramendi E, Irusta U, Ayala U, Naas H, Kramer-Johansen J, Eftest $\varnothing 1$ T. Filtering mechanical chest compression artefacts from out-of-hospital cardiac arrest data. Resuscitation 2016;98:41-47.

[4] Wik L, Olsen JA, Persse D, Sterz F, Lozano Jr M, Brouwer MA, Westfall M, Souders CM, Malzer R, van Grunsven PM, et al. Manual vs. integrated automatic load-distributing band CPR with equal survival after out of hospital cardiac arrest. The randomized CIRC trial. Resuscitation 2014; 85(6):741-748.

[5] Rubertsson S, Lindgren E, Smekal D, Östlund O, Silfverstolpe J, Lichtveld RA, Boomars R, Ahlstedt B, Skoog G, Kastberg R, et al. Mechanical chest compressions and simultaneous defibrillation vs conventional cardiopulmonary resuscitation in out-of-hospital cardiac arrest: the LINC randomized trial. Jama 2014;311(1):53-61.

[6] Isasi I, Irusta U, Elola A, Aramendi E, Ayala U, Alonso E, Kramer-Johansen J, Eftest $\varnothing 1$ T. A machine learning shock decision algorithm for use during piston-driven chest compressions. IEEE Transactions on Biomedical Engineering 2018;66(6):1752-1760.

[7] Picon A, Irusta U, Álvarez-Gila A, Aramendi E, AlonsoAtienza F, Figuera C, Ayala U, Garrote E, Wik L, KramerJohansen J, et al. Mixed convolutional and long short-term memory network for the detection of lethal ventricular arrhythmia. PloS One 2019;14(5):e0216756.

[8] Acharya UR, Fujita H, Lih OS, Hagiwara Y, Tan JH, Adam M. Automated detection of arrhythmias using different intervals of tachycardia ECG segments with convolutional neural network. Information Sciences 2017;405:81-90.

[9] Krasteva V, Ménétré S, Didon JP, Jekova I. Fully convolutional deep neural networks with optimized hyperparameters for detection of shockable and non-shockable rhythms. Sensors 2020;20(10):2875.

[10] Jaureguibeitia X, Zubia G, Irusta U, Aramendi E, Chicote B, Alonso D, Larrea A, Corcuera C. Shock decision algorithms for automated external defibrillators based on convolutional networks. IEEE Access 2020;8:154746-154758.

[11] Isasi I, Irusta U, Aramendi E, Eftest $\varnothing 1$ T, Kramer-Johansen J, Wik L. Rhythm analysis during cardiopulmonary resuscitation using convolutional neural networks. Entropy 2020; 22(6):595.

[12] Xiao Y, Ma L, Ward RK. Fast RLS Fourier analyzers capable of accommodating frequency mismatch. Signal Processing 2007;87(9):2197-2212.

[13] Isasi I, Irusta U, Aramendi E, Olsen JÅ, Wik L. Removing mechanical chest compression artefacts induced by a load distributing band device from the ECG. Resuscitation 2018; 130:e41.

Address for correspondence:

Name: Iraia Isasi Liñero

Full postal address: Ingeniero Torres quevedo Plaza, 1,Bilbao,Spain

E-mail address: iraia.isasi@ehu.eus 